\title{
Estado actual de la calidad ambiental del municipio de Turbo, Antioquia: Problemas de contaminación y alternativas de solución
}

\section{Current state of the environmental quality of the municipality of Turbo, Antioquia: Pollution problems and alternative solutions}

\section{YeneCith TORRES*}

\section{RESUMEN}

Se llevó a cabo recopilación de información en instituciones del sector público y privado, y recorridos de campo y entrevistas, con el objeto de establecer el estado actual de la calidad ambiental del municipio de Turbo. El análisis de la información muestra que el desarrollo de actividades domésticas, agroindustriales, extractivas y portuarias en el municipio y sus alrededores, generan principalmente, materia orgánica reflejada en altos valores de coliformes fecales; plaguicidas e hidrocarburos causantes de envenenamiento progresivo en la cadena alimenticia de organismos acuáticos y sólidos suspendidos que facilitan la movilidad de otros contaminantes. Esta situación, sumada a aumento de la población desplazada, a bajas coberturas de servicios públicos, y condiciones demográficas y climáticas del municipio, ha generado niveles preocupantes de contaminación revelada en una alta cantidad de personas atendidas por enfermedades gastrointestinales e infecciones respiratorias y promueve una considerable proliferación de mosquitos transmisores de enfermedades como paludismo y dengue, lo que amerita la intervención adecuada de las instituciones con influencia en el municipio, la inclusión de la comunidad en el desarrollo de investigaciones y la formulación e implementación de planes de manejo ambiental consecuentes con la realidad socioeconómica y cultural de los habitantes del municipio de Turbo.

Palabras clave: Calidad Ambiental; Problemas de contaminación;

Morbilidad y ambiente.

\begin{abstract}
Was carried out data collection in public and private institutions, field observations and interviews in order to establish the current state of environmental quality in the municipality of Turbo. The data analysis shows that the development of activities, domestic work, agro, extractive and port in the municipality and its surroundings, generated mainly organic matter reflected in high levels offecal coliform, pesticides and hydrocarbons cause progressive poisoning the food chain of aquatic organisms. This situation, coupled to the increase in the displaced population, the low coverage of public services and demographic and climatic conditions of the municipality, has generated worrisome levels of contamination revealed in the high number of people served by gastrointestinal and respiratory infections, and promotes a high proliferation of mosquitoes diseases-carrying such as malaria and dengue. Which warrants appropriate intervention influential institutions in the municipality, including the community in the development of research and the formulation and implementation of environmental management plans consistent with the
\end{abstract}

\author{
Investigadora de Proyectos Especiales, \\ Instituto de Investigaciones Ambientales \\ del Pacífico (IIAP), Quibdó, Colombia. \\ e-mail: ytorres@iiap.org.co \\ Recibido: 14 de junio de 2011 \\ Aceptado: 20 de octubre de 2011
}


socioeconomic and cultural reality of the inhabitants of the municipality of Turbo.

Keyword: Environmental quality; Environmental pollution; Disease and environment.

\section{INTRODUCCIÓN}

Calidad del agua y del aire, biodiversidad, manejo de residuos sólidos y control de vectores, son los componentes de la calidad ambiental (Garza 1998), que es definida por el MINAM (2010), como el conjunto de características del medio ambiente, relacionado con la disponibilidad y facilidad de acceso a los recursos naturales y a la ausencia de agentes nocivos, todo esto necesario para el mejoramiento de la calidad de vida de los seres humanos.

Sin embargo, aunque la calidad ambiental es considerada como una condición necesaria para el bienestar de los individuos y sociedades, rara vez se extiende más allá de esta afirmación (Kilbourne 2006), por lo que gran parte de los estudios tienden a considerar solo uno de sus componentes (Acurio 1998; Korc 2000; Herrera y Súarez 2005; Ballester 2005; Martínez-Bastida et al. 2006; Gaitán et al. 2007) y con poca frecuencia se integran todos los elementos que la caracterizan.

En este sentido, vale la pena destacar los aportes de Cabrera et al. (2002), quienes establecen las relaciones de calidad ambiental y calidad de vida en Lima, Perú, a través de la evaluación de indicadores ambientales y socioeconómicos; García et al. (2004), que propusieron indicadores basados en la integración de residuos sólidos, calidad atmosférica y del agua en la Habana, Cuba y Celemín (2007) quien estableció la asociación entre medio ambiente y calidad de vida en Mar del Plata, Argentina, a partir de una exhaustiva revisión bibliográfica que permitió conocer sus orígenes e importancia.

Para el caso de Colombia, existen dos experiencias relevantes; la primera hace referencia al Sistema de Indicadores de Calidad Ambiental, en el que se define un conjunto de 34 indicadores ambientales como un componente del desarrollo sostenible (Colnodo 2001) y la segunda incluye indicadores ambientales desarrollados en el marco de los denominados Observatorios Ambientales Urbanos (OAU), en el que se han definido 78 indicadores simples agrupados en 11 áreas temáticas, para ciudades como Armenia, Barranquilla, Bogotá, Bucaramanga, Cali, Manizales, Medellín y Pereira (RDS 2011).

No obstante para Turbo, un municipio con ubicación estratégica en la subregión del Urabá en el departamento de Antioquia, que durante décadas ha centrado su economía en la producción y comercio del banano, actividad que ha generado importantes utilidades a la economía colombiana
(Keep 2009), no se han desarrollado estudios que a través de la integración de los componentes de la calidad ambiental, permitan determinar y hacer un análisis completo y minucioso de la calidad ambiental del municipio.

En concordancia con lo anterior, la compilación, la integración y el análisis de investigaciones en la temática ambiental y de información primaria en el municipio de Turbo, obedece a la necesidad de identificar los principales contaminantes, el grado de afección que han generado las actividades económicas desarrolladas en el municipio y determinar cómo estos inciden de manera directa o indirecta en la morbilidad de sus pobladores; información necesaria para la identificación de vacíos de información, articulación de líneas de acción y ejecución de planes integrales de manejo que redunden en la protección no solo de los recursos naturales sino de las poblaciones humanas asentadas en dicho territorio.

Descripción del área de estudio. El municipio de Turbo se encuentra ubicado en la subregión de Urabá del departamento de Antioquia ( $\left.08^{\circ} 05^{\prime} 42^{\prime \prime} \mathrm{N}, 76^{\circ} 44^{\prime} 23^{\prime \prime} \mathrm{O}\right)$. Limita al norte con el mar Caribe y los municipios de Necoclí y Arboletes; al este con los municipios de San Pedro de Urabá, Carepa, Chigorodó y Apartadó y con el departamento de Córdoba; al sur con el municipio de Mutatá y al oeste con los municipios de Riosucio y Ungía en el departamento del Chocó (Mapa 1). Tiene una extensión de $3055 \mathrm{~km}^{2}$, de los cuales $11,9 \mathrm{~km}^{2}$ hacen parte del área urbana y $3043 \mathrm{~km}^{2}$, al área rural (POT municipio de Turbo 2000).

Está bañado por los ríos Atrato, León, Turbo, Guadualito, Currulao, Grande y Caimán Nuevo (POT Municipio de Turbo 2000), de donde el río Atrato representa una fuente hídrica de gran valor ecológico, social y económico para el municipio (CORPOURABÁ) 2002). Cuenta con una población de 121.919 habitantes, de los cuales el 44,1\% gozan del servicio de acueducto, el 30,7\% de alcantarillado y el 51,8\% del servicio de recolección de residuos sólidos (DANE 2005).

Presenta una economía eminentemente agropecuaria centrada principalmente en la producción y comercio de banano, actividad que genera la mayor cantidad de empleos, puesto que productos como arroz, cacao y maíz son aún marginales en la actividad productiva (Cámara de Comercio 2005). La extracción forestal y la pesca, aunque tampoco son actividades de relevancia económica en la economía del municipio, han sido durante décadas, una fuente importante de ingresos para la población (Taborda et al. 2008).

\section{MÉTODOS}

Se entrevistó a profesionales encargados de diferentes temáticas ambientales en la Corporación Autónoma para el Desarrollo Sostenible del Urabá (CORPOURABÁ) y otros 
Bioetnia Volumen 8 № 2 (julio-diciembre), 2011

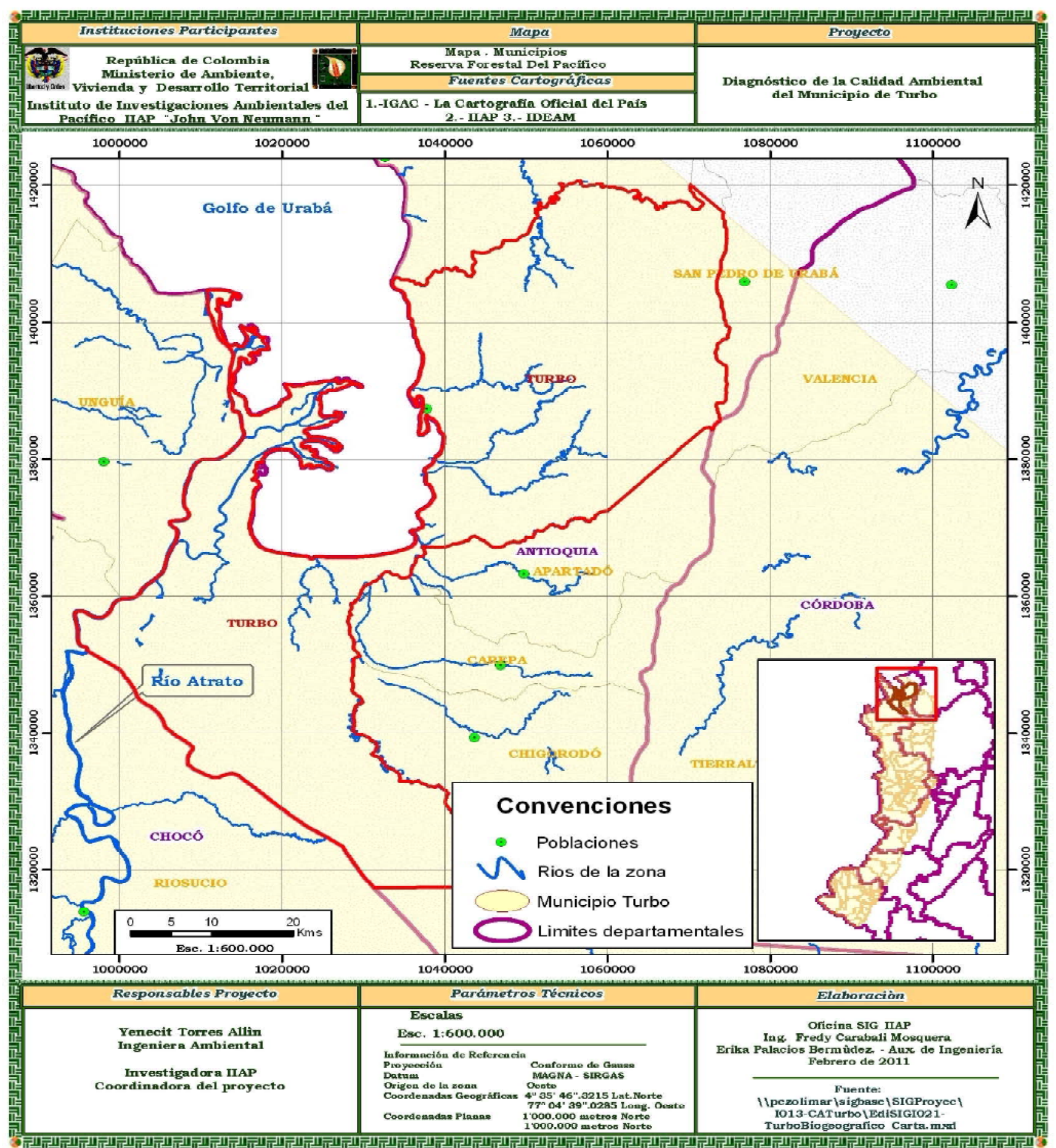

Mapa 1. Ubicación Geográfica del Municipio de Turbo

investigadores del municipio, quienes permitieron obtener una noción preliminar de los trabajos desarrollados y los vacíos de información en la temática de estudio. Se consideró la zona centro, los barrios Manizales y La Lucila, y los corregimientos El Dos, El Tres y Nueva Granada, para el desarrollo de recorridos y entrevistas no estructuradas a sus habitantes, sectores sugeridos por el autor de Monografías de Antioquia (2009), Fernando Keep, basado en factores como insuficiencia en cobertura de servicios públicos y alta presencia de población desplazada. 
En los centros de documentación de CORPOURABÁ, Universidad de Antioquia, Asociación de Bananeros de Colombia (AUGURA), Alcaldía municipal y otras instituciones con influencia en el municipio, se llevó a cabo una revisión bibliográfica exhaustiva de todos los documentos relacionados con las temáticas calidad del agua, del aire, residuos sólidos y biodiversidad. En la web, se realizó una búsqueda de todas las investigaciones relacionadas con calidad ambiental y calidad de vida, llevadas a cabo en diferentes lugares del mundo y específicamente en el municipio de Turbo, y en la página web del Instituto de Investigaciones Marinas y Costeras (INVEMAR) se obtuvieron los diagnósticos de calidad ambiental de aguas marinas y costeras en el Caribe y Pacífico colombiano desarrollados entre los años 2001 y 2010.

A la sección de estadística del Hospital Francisco Valderrama, se hizo solicitud escrita del informe de vigilancia epidemiológica del municipio de Turbo para los años 2002-2009 (datos sin publicar) y al Instituto de Meteorología e Hidrología (IDEAM, datos sin publicar) se solicitó información sobre pluviosidades entre los mismos años, datos que fueron facilitados por ambas instituciones

Con el objeto de obtener información precisa sobre diferentes componentes de la calidad ambiental en el municipio de Turbo, considerando las recomendaciones para la elaboración de artículos de revisión citadas por Cué et al. (1996) y Guirao-Goris et al. (2008), se llevó a cabo una revisión minuciosa de todos los documentos encontrados y/ o facilitados, donde se desecharon los considerados irrelevantes y se seleccionaron solo aquellos que proporcionaban datos útiles y confiables, es decir, pertinentes al tema y sin deficiencias metodológicas notorias. Del informe de Vigilancia Epidemiológica solo se tuvieron en cuenta las patologías relacionadas directamente con calidad ambiental y saneamiento básico como es el caso de paludismo, dengue, enfermedades diarreicas agudas (EDA) e infecciones respiratorias agudas (IRA). La información considerada pertinente se combinó de la mejor manera posible y se analizó mediante la integración selectiva y sistemática de los resultados.

\section{RESULTADOS Y DISCUSIÓN}

La calidad ambiental del municipio de Turbo ha disminuido a través de los años y a pesar de la implementación de algunas alternativas de solución, a la fecha presenta niveles preocupantes de contaminación, ligadas principalmente a una explotación inadecuada de recursos naturales, insuficiente cobertura de servicios públicos, y procesos agroindustriales y de urbanización sin criterios de planificación.
El problema progresivo de contaminación ambiental. Las condiciones ambientales iniciales del municipio de Turbo se analizan en estudios desarrollados por Ramírez (1981) hace 30 años cuando el río Turbo presentaba poca polución, pues cruzaba lejos de la población del mismo nombre y el desarrollo industrial de la zona era muy precario.

Los problemas de contaminación han venido aumentado sustancialmente debido a las bajas coberturas de alcantarillado y falta de operación adecuada de las plantas de tratamiento de aguas residuales del municipio de Turbo, lo que se refleja en los resultados del análisis de calidad de aguas del río Turbo en algunos sectores de la zona urbana como son el caño Veranillo, bahía de Turbo y el Waffe, donde se muestran altos niveles de contaminación por coliformes fecales con valores que alcanzan los $22.000 \mathrm{NMP} / 100 \mathrm{ml}$, sobrepasando los límites permisibles para natación, buceo y pesca (decreto 1594/84); estos resultados muestran además, bajos valores de oxígeno disuelto (hasta $0,5 \mathrm{mg} / \mathrm{l}$ ) y altas demandas bioquímicas (201,5 mg/l) (CORPOURABÁ 2007a); en adición, los índices de calidad ambiental (ICA) para esa misma época (CORPOURABÁ 2007b), comparados con los calculados por CORPOURABÁ (2009a), reflejan de mejor manera el deterioro progresivo de la calidad ambiental del río Turbo (Figura 1), situación revelada en los 10.902 pacientes atendidos por EDA por el Hospital Francisco Valderrama entre los años 2005, 2007 y 2008, siendo 2008 con 4191 casos, el año con mayor reporte (Figura 2).

Como todos los cuerpos de agua dulce que atraviesan el municipio de Turbo desembocan en el mar Caribe, el escenario en aguas marino costeras es aún más preocupante. Los resultados de Troncoso et al. (2008), revelan altos aportes de coliformes totales y fecales en las desembocaduras de algunos ríos del municipio, donde los puntos que han registrado mayores aportes de coliformes totales entre 2001 y 2007 son León, Mulatos (4.600.000 NMP/100ml), Currulao (430.000 $\mathrm{NMP} / 100 \mathrm{ml}$ ) y Turbo (160.000 NMP/100 ml); para 2008, la playa de Turbo reportó el máximo valor de coliformes fecales registrado en los últimos 10 años en los puntos establecidos en el Caribe con 15.000 NMP/100 ml; todo lo anterior atribuido principalmente a aumentos en las concentraciones de materia orgánica, producto de las actividades agroindustriales, derivadas del cultivo del banano, principal actividad económica del municipio.

La producción del banano requiere de la utilización de grandes cantidades de plaguicidas; históricamente se registran concentraciones preocupantes de plaguicidas organoclorados en el río Turbo con valores de $9 \mathrm{ng} / \mathrm{l}$ (Garay et al. 2004), el río Atrato en su desembocadura con 15,9 ng/l (Garay et al. 2005) y la desembocadura del río León con 42,7 ng/l, donde se reporta la concentración histórica más alta en la región (Troncoso et al. 2007), superando los $30 \mathrm{ng} / \mathrm{l}$ 
Bioetnia Volumen 8 No 2 (julio-diciembre), 2011
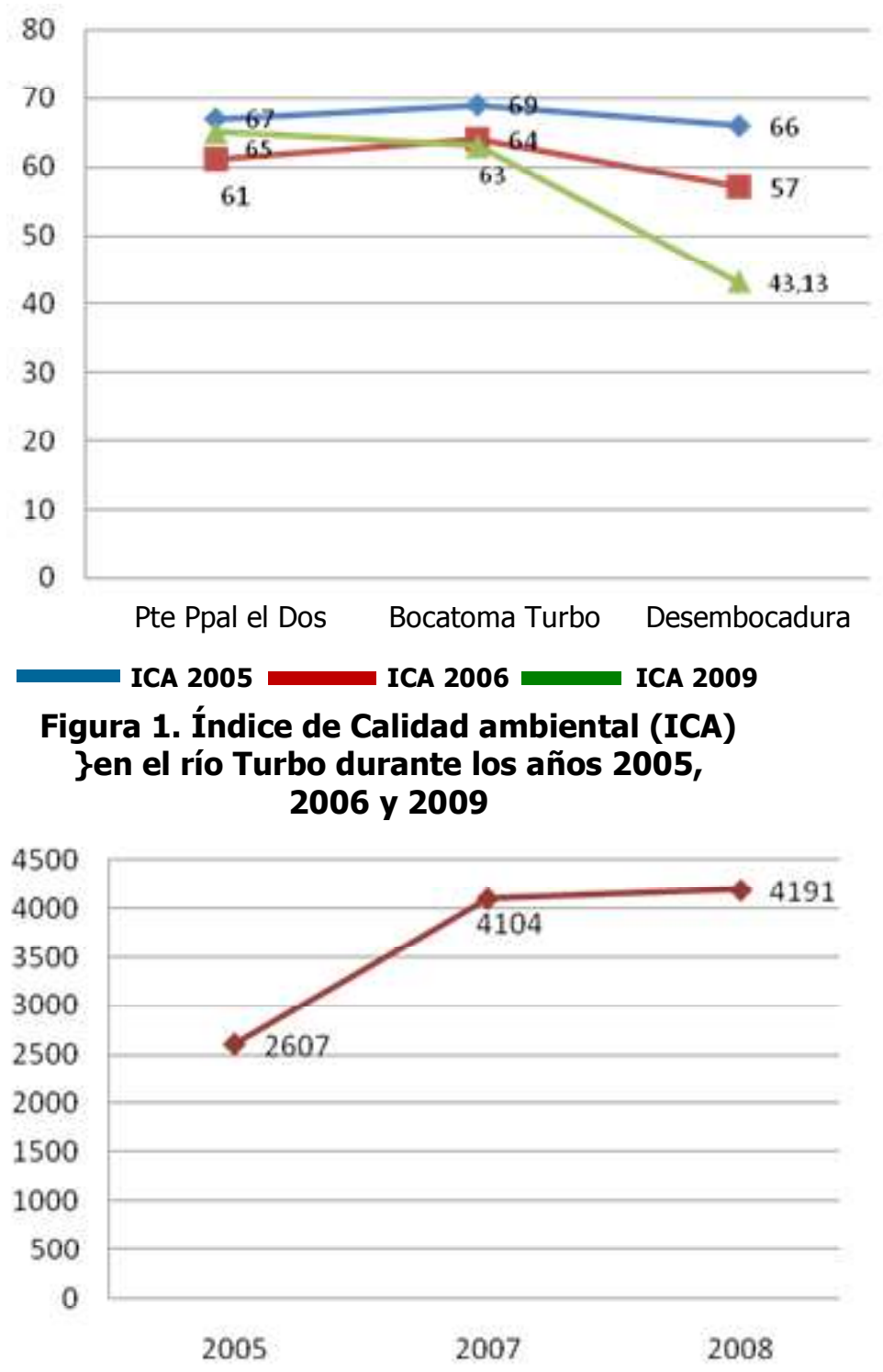

Figura 2. Aumento progresivo de casos de EDA en el municipio de Turbo entre los años 2005, 2007 y 2008

establecidos como valor límite a nivel nacional para actividades recreativas y pesca (Decreto 1594/84).

Adicionalmente, la utilización de grandes embarcaciones para el transporte marítimo del banano y el movimiento de pequeñas embarcaciones dedicadas al comercio, transporte de personas y el turismo, generan concentraciones de hidrocarburos $(\mathrm{HC})$ que en ocasiones han superado los $10 \mu \mathrm{g} / \mathrm{l}$, establecidos como valor límite para aguas marinas y costeras (UNESCO 1984; Atwood et al. 1988), como es el caso de la desembocadura del río León, donde los valores sobrepasaron los $15 \mu \mathrm{g} / 1$ (Garay et al. 2001). La desembocadura del río Mulatos ha presentado niveles máximos de $4.6 \mu \mathrm{g} / 1$ (Troncoso et al. 2007), que si bien no corresponden a valores que puedan considerarse contaminantes, han sido de particular atención. En la actualidad, a pesar de los esfuerzos de la autoridad ambiental para evitar el uso de estos contaminantes, se observa una presencia recurrente de plaguicidas organoclorados en las desembocaduras de los ríos Turbo y León (Troncoso et al. 2009), hecho que representa graves riesgos para la permanencia de más de 60 especies de peces de gran valor económico para los habitantes del municipio (CORPOURABÁ et al. 2005).

Estos riesgos para la biota fueron también detectados por Mejía y Gómez (2002), quienes estimaron la existencia de graves indicios de contaminación y alteración de las cuencas del municipio, atribuidos principalmente a la deforestación que genera aporte de sedimentos que facilitan la movilización de los diferentes contaminantes en aguas marino costeras y continentales, y que de acuerdo con las anotaciones de Fonde-CORPOURABÁ (2002), es causada por colonos y nativos que dependen de la corta del mangle para su sostenimiento económico, hecho que atenta contra la diversidad de especies de mamíferos reportadas en manglares por Sánchez-Paezy ÁlvarezLeón (2003) y especies típicas de mangle como es el caso de Rizophora mangle (mangle rojo), Laguncularia racemosa (mangle bobo) (CORPOURABÁ et al. 2005).

Recientemente, el alto aporte de sedimentos se evidencia en los valores de sólidos suspendidos totales que alcanzan $884 \mathrm{mg} / \mathrm{l}$ y $544 \mathrm{mg} / \mathrm{l}$ en las desembocaduras de los ríos Turbo y Mulatos respectivamente (Troncoso et al. 2008).

Las situaciones anteriores se tornan aún más críticas por las bajas coberturas en recolección de residuos sólidos, barrido de vías y áreas públicas llevadas a cabo en menos del $20 \%$ del municipio, que ligadas a la falta de campañas de educación ambiental para el manejo adecuado de residuos sólidos y tratamiento de otros problemas ambientales, hacen notoria la gran cantidad de residuos en las aguas, calles y playas del municipio, principalmente en sectores registrados en la zona urbana por CESID (2005), como son El Wafe, Plaza de Mercado, Calle 101, Buenos Aires, La Playa, Caño Puerto Tranquila, barrio El Bosque y Ciudadela Bolívar.

Los problemas de salubridad se reflejan en 742 casos de dengue clásico y hemorrágico y 26.168 casos de paludismo reportados por el Hospital Francisco Valderrama entre los años 2002-2009, que se relacionan directamente con los niveles anuales de lluvia (Figura 3); en este sentido Carmona-Fonseca (2003) aseguran que para el caso específico del paludismo, su presencia obedece no solo a condiciones climáticas sino demográficas y socioeconómicas, 


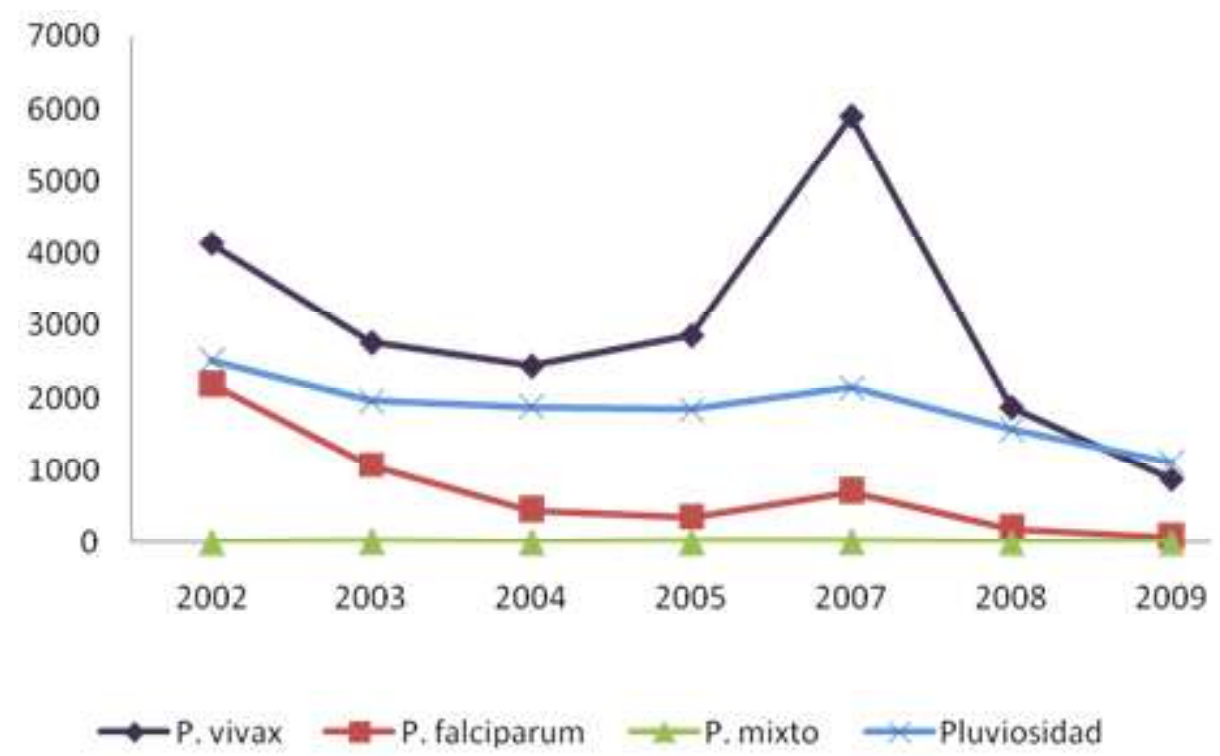

Figura 3. Casos de malaria según la especie de Plasmodium en el municipio de Turbo entre los años 2002-2005 y 2007-2009 y su relación con la pluviosidad

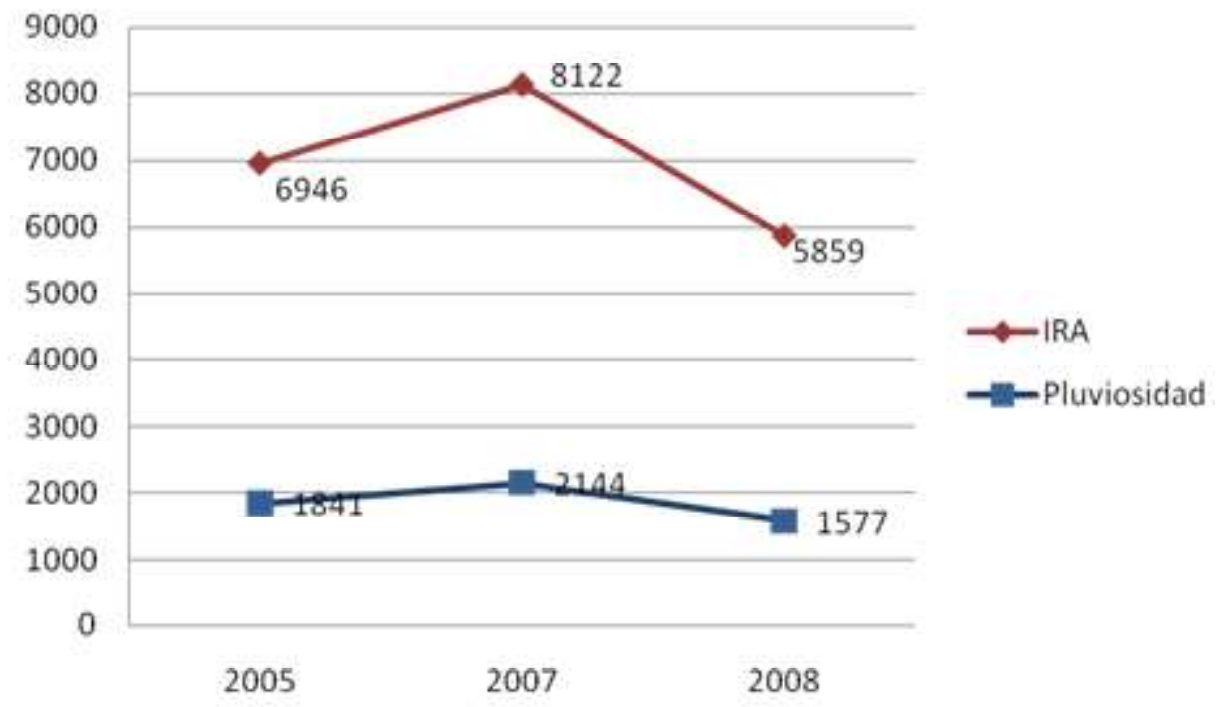

Figura 4. Casos de IRA en el municipio de Turbo entre los años 2005, 2007 y 2008 y su relación con la pluviosidad

y se tornan aún más críticas por el aumento de la población desplazada del municipio de Turbo, que de acuerdo con Bastidas et al. (2010) asciende a 20.986 personas, correspondientes al 96\% del total del departamento de Antioquia.

Por otra parte, los procesos de urbanización sin criterios de planificación del municipio de Turbo, vienen alterando su calidad atmosférica. CORPOURABÁ (2009b) registra niveles preocupantes de ruido que superan en el 94\% de las mediciones los valores estipulados por la Resolución 0627 de 2006, en el período nocturno por el uso indiscriminado de equipos de sonido pickups $[83 \mathrm{~dB}(\mathrm{~A})] \mathrm{y}$ en el período diurno por el alto tráfico vehicular $[70 \mathrm{~dB}(\mathrm{~A})]$, porque la Secretaría de Tránsito Municipal registra para 2010 un total de 9753 motocicletas, 500 carros particulares y 700 carros públicos, vehículos que si no reciben el mantenimiento oportuno, por las emisiones de gas carbónico (CO) e hidrocarburos (HC) podrían estar contaminando el ambiente y a su vez generarían graves afecciones respiratorias a la población.

En este sentido, el Hospital Francisco Valderrama informa 20.927 casos de IRA entre los años 2005, 2007 y 2008, 


\section{Bioetnia Volumen 8 No 2 (julio-diciembre), 2011}

siendo 2007 el año de mayor pluviosidad y con mayor registro de pacientes atendidos (Figura 4). En adición, para 2009, 24 niños menores de cuatro años murieron por IRA, situación que amerita atención prioritaria de las entidades de salubridad ambiental del municipio.

Alternativas de solución para el mejoramiento de la calidad ambiental. En la actualidad, las instituciones con influencia en el municipio de Turbo, pese a sus notables esfuerzos, no ejecutan acciones suficientes para contrarrestar las presiones ejercidas sobre el ambiente. Esto se ratifica con la explotación irracional que aún se hace de los recursos naturales, la gran cantidad de residuos sólidos observadas en calles y playas, el uso indiscriminado de equipos de sonido pickups, el alto tránsito vehicular en zonas urbanas no permitidas y la presencia recurrente de plaguicidas organoclorados en los cuerpos de agua; a pesar de las actividades desarrolladas en torno a la declaración de zonas de recuperación de ecosistemas estratégicos (INVEMAR et al. 2007), al desarrollar actividades de capacitación y la sensibilización de los habitantes (Geerders et al. 2008) y campañas para reducción de agroquímicos (Troncoso et al. 2009).

En este sentido, se hace necesario propender por un ordenamiento territorial que reconozca el patrimonio urbano, rural y paisajístico del municipio de Turbo, tendiente a lograr una ciudad moderna, atractiva y acogedora. En otras palabras esto significa:

- Una cobertura de acueducto de agua potable que llegue al $100 \%$ de los turbeños. Aunque la principal fuente de agua empleada para la prestación del servicio de acueducto es el río Turbo, que además de presentar problemas de contaminación, en épocas de verano intenso no alcanza a cubrir los caudales mínimos (200 1/seg) necesarios. Villegas y Vivas (2009), reportan la existencia de 137 pozos de aguas subterráneas, de buena calidad (CORPOURABÁ, 2009c), que de acuerdo con estudios desarrollados por la Universidad Nacional (2010) pueden ser combinadas con las fuentes de agua superficial para el suministro de suficiente agua potable.

- Una cobertura de alcantarillado para el 100\% de la población, considerando además la construcción y funcionamiento de plantas de tratamiento de aguas residuales en la subregión del Urabá.

- Gestionar integralmente los residuos sólidos del municipio y territorios aledaños, ligado con el control en sectores precarios, del riesgo sanitario que generan los mosquitos y otras plagas.

- Establecer y aplicar medidas de conservación, manejo y utilización sustentable de los recursos naturales.

- Controlar la contaminación acústica proveniente de fuentes fijas y móviles de acuerdo con las normas de emisión vigentes.

- Generar, a través de la educación ambiental y participa- ción comunitaria, cambios culturales para la prevención del deterioro ambiental.

En adición, se requiere de la articulación adecuada entre diferentes instituciones con influencia en el territorio, lo cual evitaría la duplicación de información y permitiría el desarrollo de estudios, normas y obras pertinentes y tendientes a la conservación y protección de los recursos naturales.

\section{CONCLUSIONES}

Turbo es un municipio rico en especies de flora y fauna, recursos hídricos y agrícolas, con múltiples beneficios otorgados por su ubicación geográfica, con lo que puede garantizar un desarrollo adecuado y un bienestar colectivo. Sin embargo, se presentan problemáticas sociales y administrativas relacionadas con la educación, la salud y la sanidad, que amenaza la subsistencia de especies de flora y fauna, sustento económico de muchos turbeños. Además, viene disminuyendo la calidad y cantidad de los recursos hídricos del municipio y genera enfermedades que afectan sobre todo a la población infantil. Esta situación amerita el desarrollo de intervenciones donde se articulen las instituciones con pertinencia en el tema ambiental y de la formulación e implementación de un plan de ordenamiento territorial y de planes de manejo, consecuentes con sus recursos naturales y con la realidad socioeconómica y cultural de sus habitantes.

\section{AGRADECIMIENTOS}

La elaboración de este trabajo se llevó a cabo gracias a información facilitada por CORPOURABÁ, Universidad de Antioquia, AUGURA, Hospital Francisco Valderrama y Alcaldía Municipal, y al apoyo técnico y humano del Instituto de Investigaciones Ambientales del Pacífico (IIAP), Quibdó.

\section{LITERATURA CITADA}

Acurio, G., A. Rossi, P. Teixera, F. Cepeda. 1998. Diagnóstico de la situación del manejo de residuos sólidos municipales en América Latina y el Caribe. Banco Interamericano de Desarrollo y Organización Panamericana de la Salud. Septiembre de 1998. Serie Ambiental $N^{\circ} 18$. En línea [14 de agosto de 2011]. URL disponible en: http://www.bvsde.opsoms.org/cdrom-repi86/fulltexts/bvsacd/scan/dsm.pdf

Atwood D. K., F. J. Burton, J. E. Corredor, G. R. Harvey, A. J. Mata-Jiménez, A. Vasquez-Botello, et al. 1988. Petroleum Pollution in the Caribbean Oceanus. Caribbean Environmental Health Institute. Marine Polut Bull. 30 (4): $25-32$.

Bastidas, W., N. Espinosa, M. Piedrahita, A. Galindo, J. E. Monsalve,Y. Lopera. 2010. Informe de caracterización de la población en situación de desplazamiento del municipio de Turbo. ACNUR, Gobernación de Antioquia, Observatorio del Desplazamiento Forzado, Comité para la Atención Integral a Desplazados de Antioquia. 13 pág. En línea [14 de febrero de 2011]. URL disponible en: http://www.comitedesplazados antioquia. gov.co/Observatorio/Caracterizaciones/TURBO.pdf

Ballester, F. 2005. Vigilancia de riesgos ambientales en Salud Pública: El caso 